over 50 than those under 30 , but their cost rates were only modestly higher.

Conclusions Progress has been made in reducing direct costs associated with FFH in this cohort particularly though 1996, but primary gains reflect the reduction in frequency while FFH that occur remain serious.

\section{PESTICIDE EXPOSURE DURING RE-ENTRY TASKS AND HARVESTING IN VINEYARDS: RESULTS OF THE PESTEXPO PROGRAM}

1,2I Isabelle Baldi, ${ }^{3,4}$ Pierre Lebailly, ${ }^{1}$ Ghislaine Bouvier, ${ }^{1}$ Virginie Rondeau, ${ }^{5}$ Valérie Bouchart, ${ }^{1}$ Mireille Canal-Raffin, ${ }^{1}$ Alain Garrigou. ${ }^{1}$ Univ. Bordeaux, ISPED, Laboratoire Santé Travail Environnement, Centre INSERM U 897 Epidemiologie-Biostatistique, F-33000 Bordeaux, France; ${ }^{2}$ CHU de Bordeaux, Service de Médecine Du Travail, F-33000 Bordeaux, France; ${ }^{3}$ INSERM, UMR 1086-Cancers Et Préventions, F-14000 Caen, France; ${ }^{4}$ Centre François Baclesse, F-14000 Caen, France; ${ }^{5}$ Laboratoire Départemental Frank Duncombe, F-14000 Caen, France

\subsection{6/oemed-2014-102362.129}

Objectives Physical contact with treated crops, animals or other surfaces is responsible for the transfer of pesticides to the worker's skin in agricultural tasks and makes their cutaneous absorption possible. In the Bordeaux area (France), the PESTEXPO study described levels of pesticide exposure and identified their determinants during re-entry and harvesting in vineyards.

Method Between 2002 and 2007, 46 days of work involving reentry tasks and 48 harvesting days were observed to analyse exposures to dithiocarbamates or folpet. The potential determinants were generated from the following parameters collected on standardised forms during field observations: i)general conditions of the task, ii)operator characteristics, iii)estate characteristics, iv) task conditions and v)characteristics of the last treatment involving folpet or dithiocarbamates, including delay since treatment. Dermal contamination was assessed using patches placed on the skin and hand-washing at the end of each working phase. Results Daily median contamination was $1967.7 \mu \mathrm{l}$ of mixture during re-entry (90e percentile: $5045.3 \mu \mathrm{l}$ ) and $18.7 \mu \mathrm{l}$ during harvesting $(90 \mathrm{e}$ percentile: $911.4 \mu \mathrm{l})$. Contamination level was strongly correlated to the type of task. For re-entry, the highest contaminations were observed during raising of wires and cutting of branches. During the harvest, the contamination was maximal for grape-picking. The delay since the last treatment and the rate of active ingredient per hectare played a role, together with meteorological factors, crop and farm characteristics, gloves and clothes.

Conclusions Our results underline the necessity to take into account exposures during re-entry and harvest when considering pesticide exposure, both for epidemiological research and preventive action.

\section{OCCUPATIONAL EXPOSURE TO LEAD AND CANCER IN TWO COHORT STUDIES OF MEN AND WOMEN IN SHANGHAI, CHINA}

${ }^{1}$ Linda M Liao, ${ }^{1}$ Melissa C Friesen, ${ }^{2}$ Yong-Bing Xiang, ${ }^{3}$ Hui Cai, ${ }^{4}$ Dong-Hee Koh, ${ }^{1}$ Bu-Tian Ji, ${ }^{3}$ Gong Yang, ${ }^{2}$ Hong-Lan Li, ${ }^{1}$ Sarah J Locke, ${ }^{1}$ Nathaniel Rothman, ${ }^{3}$ Wei Zheng, ${ }^{2}$ Yu-Tang Gao, ${ }^{3}$ Xiao-Ou Shu, ${ }^{1}$ Mark P Purdue. ${ }^{1}$ Division of Cancer Epidemiology and Genetics, National Cancer Institute, Bethesda, MD, USA; ${ }^{2}$ Department of Epidemiology, Shanghai Cancer Institute, Shanghai, China; ${ }^{3}$ Department of Medicine, Vanderbilt University, School of Medicine, Nashville, TN, USA; ${ }^{4}$ Carcinogenic Hazard Branch, National Cancer Center, Seoul, Republic of Korea

10.1136/oemed-2014-102362.130
Objectives Epidemiologic studies of occupational lead exposure have suggested increased risks of cancers of the brain, kidney, lung, meninges, and stomach; however, the totality of the evidence is inconsistent. To clarify whether lead is a carcinogen, we investigated the relationship between occupational lead exposure and risks of these five cancer sites in two prospective cohort studies in Shanghai, China.

Method Annual job/industry-specific estimates of lead fume and lead dust exposure were derived from a statistical model that combined expert ratings of lead intensity with inspection measurements collected by the Shanghai Centre for Disease Control and Prevention. The job/industry estimates were applied to the lifetime work histories of subjects from the Shanghai Women's Health Study (73 363 participants) and the Shanghai Men's Health Study (61 379 participants) to estimate cumulative exposure to lead dust and lead fume. Cohort-specific relative hazard rate ratios (RRs) and 95\% confidence intervals (CI) were estimated using Cox proportional hazards regression models and then pooled using a random effects meta-analysis model.

Results We observed a statistically significant increased risk of meningioma among individuals with estimated occupational exposure to lead dust or fumes ( $\mathrm{RR}=2.4,95 \% \mathrm{CI}: 1.1-5.0)$, and in particular among those with an above-median cumulative exposure to dust or fumes ( $R R=3.1,95 \%$ CI:1.3-7.4). We observed suggestive associations with lead exposure for cancers of the kidney ( $\mathrm{RR}=1.4,95 \% \mathrm{CI}: 0.9-2.3)$ and brain $(\mathrm{RR}=1.8$, 95\% CI:0.7-4.8), and null findings for cancers of the lung and stomach.

Conclusions Our findings provide additional evidence that occupational lead exposure increases risk of meningioma.

\section{POSSIBLE PRO-CARCINOGENIC EFFECT OF ENDOTOXIN ON LUNG CANCER IN AN EXTENDED FOLLOW-UP OF SHANGHAI WOMEN TEXTILE WORKERS}

${ }^{1}$ Harvey Checkoway, ${ }^{2}$ Jessica Lundin, ${ }^{3}$ Sadie Costello, ${ }^{4}$ Roberta Ray, ${ }^{4}$ Wenjin Li, ${ }^{3}$ Ellen Eisen, ${ }^{5}$ George Astrakianakis, ${ }^{2}$ Noah Seixas, ${ }^{6}$ Kate Applebaum, ${ }^{7}$ Dao Li Gao, ${ }^{4}$ David Thomas. ${ }^{1}$ University of California, San Diego, La Jolla, CA, USA; ${ }^{2}$ University of Washington, Seattle, WA, USA; ${ }^{3}$ University of California, Berkeley, Berkeley, CA, USA; ${ }^{4}$ Fred Hutchinson Cancer Research Center, Seattle, WA, USA; ${ }^{5}$ University of British Columbia, Vancouver, BC, Canada; ${ }^{6}$ George Washington University, Washington, DC, USA; 'Zhong Shan Hospital, Shanghai, China

\subsection{6/oemed-2014-102362.131}

Objectives To examine further the association between endotoxin and risk of lung cancer among Shanghai women textile workers in an extended follow-up of the cohort. The initial follow-up indicated an inverse exposure-response relation.

Method We updated a case-cohort study nested within a cohort of 267400 women. We compared exposure histories of 1456 incident lung cancers cases diagnosed during 1989-2006 with those of a reference subcohort of 3022 workers who were free of lung cancer at the end of follow-up. Endotoxin exposures were based on a quantitative job/exposure matrix. Relative risks (hazard ratios [HR]) associated with cumulative exposure, adjusted for age and smoking history, were estimated by Cox proportional hazards modelling adapted for the case-cohort design. We conducted exposure-response trend analyses for cumulative exposures lagged by 0,10 , and 20 years, and separately for time windows of $<15$ and $>15$ years since first exposure.

Results Overall, we observed no associations between cumulative exposure and lung cancer. In contrast, analyses by exposure 
time windows revealed a modestly elevated risk at the highest 3 exposure quintiles for exposures that occurred $>15$ years since first exposure; $\mathrm{HR}=1.28$ (95\% CI 0.90-1.82), $\mathrm{HR}=1.27(95 \%$ CI 0.93-1.73), and HR=1.27 (95\% CI 0.91-1.77), respectively; p-trend $=0.13$.

Conclusions Exposures to endotoxin with long-term, relatively intense exposures were at most weakly associated with lung cancer risk in this cohort. The findings do not support a protective effect of endotoxin, but are suggestive of possible lung cancer promotion with increasing time since first exposure.

\section{CANCER RISK ASSESSMENT IN PEOPLE HIGHLY EXPOSED TO PCBS AND PCDFS BASED ON SERUM CONCENTRATIONS 15-24 YEARS AFTER EXPOSURE}

${ }^{1,2}$ Leon Guo, ${ }^{2,3}$ Shih-Che Hsu, ${ }^{3}$ Gen-Shuh Wang. 'Environmental and Occupational Medicine, National Taiwan University College of Medicine (NTU) and NTU Hospital, Taipei, Taiwan; ${ }^{2}$ Institute of Occupational Medicine and Industrial Hygiene, College of Public Health, National Taiwan University, Taipei, Taiwan; ${ }^{3}$ Institute of Environmental Health, College of Public Health, National Taiwan University, Taipei, Taiwan

\subsection{6/oemed-2014-102362.132}

Objectives Risk associated with dioxin-like chemicals (DLCs) can be estimated using cancer slope factor (SF) derived from epidemiology data, and lifetime average daily dose (LADD). However, for shorter term exposure, such analysis has not been done. We propose a method to estimate cancer risk using internal exposure dose.

Method In 1979, approximately 2000 people in central Taiwan accidentally consumed rice oil contaminated by dioxin-like chemicals polychlorinated biphenyls (PCBs)- and dibenzofurans (PCDFs). Blood samples were collected between 1994 and 2003. Serum toxic equivalency (TEQ) was back-extrapolated to the time at the beginning of the exposure by using half-life of 8.7 years. The LADD of the background population was estimated by serum level, and the excess cancer risk of the background population was estimated by multiplying LADD by the cancer SF. Thereafter, the LADD and excess cancer risk of the Yucheng population was estimated correspondingly by the ratio of serum TEQ area under curve (AUC) between the exposed and the background population.

Results The average serum concentration of 245 exposed people in 1994-2003 and the estimated serum concentration in 1979 were $424(\mathrm{SD}=334)$ and $1602(\mathrm{SD}=1135)$ pg-TEQ/g-lipid, respectively. The estimated LADD of DLCs in background population was $2.18 \mathrm{pg}-\mathrm{TEQ} / \mathrm{kg}$-body weight/day, and the lifetime excess cancer risk caused by background exposure to DLCs is $3.4 * 10-4$. The average value of exposed people's serum TEQ AUC and the risk (i.e., 5.7*10-3) are16.8 times higher than those of the background population.

Conclusions Based on this method, individual risk can be estimated when serum concentration of DLCs are available.

\section{NON-FATAL AGRICULTURAL INJURIES: SURVEILLANCES IN THE MIDWESTERN UNITED STATES}

1,2 Lina Lander, ${ }^{1,2}$ Ketki Patel, ${ }^{1,3}$ Dana Loomis, ${ }^{1}$ Shinobu Watanabe-Galloway, ${ }^{1}$ Gleb Haynatzki, ${ }^{1}$ Rosa Gofin, ${ }^{1,2}$ Rohan Jadhav, ${ }^{1,2}$ Risto Rautiainen. ${ }^{1}$ University of Nebraska Medical Center, Omaha, Nebraska, USA; ${ }^{2}$ Central States Center for Agricultural Safety and Health, Omaha, Nebraska, USA; ${ }^{3}$ International Agency for Research on Cancer, Lyon, France

10.1136/oemed-2014-102362.133
Objectives The objective was to estimate the agriculture injury rate in in the United States. The Bureau of Labour Statistics conducts Census of Fatal Occupational Injuries and Survey of Occupational Injuries and Illness but it excludes workplaces with 10 or fewer employees or self-owned farm operations and may underestimate the agricultural injury rate.

Method The Central States Centre of Agricultural Safety and Health partnered with National Agriculture Statistics Service to annually administer agricultural injury survey. In 2012, 6953 surveys were administered to a stratified random sample of 2007 Census of agriculture respondents in seven Midwestern States. The survey included questions on demographics, type, location and source of injury, body part injured, lost work time, and cost. The data were linked to Census of agriculture for farm level attributes. Univariate and multivariate logistic regressions were used to evaluate factors associated with adult operator injuries.

Results The cumulative incidence was 60.6 injuries per 1000 farm operators. Injury incidence was significantly higher in parttime compared to full-time farmers (79.3 vs 42.6 per 1000 , p < 0.0001); farm size 1000 or more acres compared to 180-999 and $1-179$ acres (91.6 vs. 60.5 and 45.4 per $1000, \mathrm{p}=0.002$ ); at least one livestock compared to none (77.1 vs. 44.3 per 1000 , $\mathrm{p}=0.0004)$; and having a tractor with 100 or more horsepower (71.8 per $1000, \mathrm{p}=0.006$ ).

Conclusions There were substantial differences in injury incidence by individual and farm attributes. These results may be used to develop targeted interventions to reduce agricultural injuries in the Midwestern United States.

\section{JOB EXPOSURES, HEALTH BEHAVIOURS, AND WORK- RELATED INJURIES AMONG YOUNG CONSTRUCTION WORKERS IN THE UNITED STATES: A 12-YEAR FOLLOW-UP STUDY}

Xiuwen Sue Dong, Xuanwen Wang, Julie Largay. CPWR - the Center for Construction Research and Training, Silver Spring, MD, USA

\subsection{6/oemed-2014-102362.134}

Objectives Young construction workers are at increased risk for occupational injuries. This study aimed to identify factors associated with work-related injuries within this worker group in order to provide insight for injury interventions.

Method Data from nine waves (1988-2000) of the National Longitudinal Survey of Youth, 1979 cohort (NLSY79, N=12 686), were studied. Construction workers included those who worked in the construction industry for at least one wave. Job exposures were measured by frequency and types of physical efforts, number of waves worked in blue-collar jobs, and hours worked per week. Health behaviours were composed of body mass index, and dose of tobacco, alcohol, marijuana, and cocaine use. Multivariate logistic regression was employed to estimate the association between selected factors and work-related injuries after controlling for possible confounders.

Results During the 12-year follow-up period, $47 \%$ of the construction cohort experienced work-related injuries compared to $29 \%$ for their non-construction counterparts. The logistic regression results indicated that both job exposures and personal behaviours were associated with work-related injuries: blue-collar occupations (OR $=4.24,95 \%$ CI: 2.54-7.07); physical efforts $(\mathrm{OR}=1.72,95 \% \mathrm{CI}: 1.20-2.48)$; worked over $50 \mathrm{~h}$ per week $(\mathrm{OR}=1.91,95 \% \mathrm{CI}: 1.11-3.28)$; rotating/split shift $(\mathrm{OR}=2.99,95 \% \mathrm{CI}: 1.25-7.16)$; obesity $(\mathrm{OR}=1.58,95 \% \mathrm{CI}$ : 